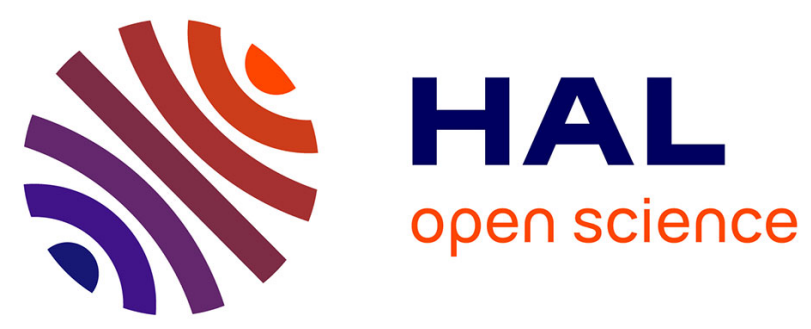

\title{
Transposing Computer Tools from the Mathematical Sciences into Teaching \\ Jean-Baptiste Lagrange
}

\section{To cite this version:}

Jean-Baptiste Lagrange. Transposing Computer Tools from the Mathematical Sciences into Teaching: Some possible obstacles. The Didactical Challenge of Symbolic Calculators Turning a Computational Device into a Mathematical Instrument, 36, Springer-Verlag, 2005, Mathematics Education Library, 10.1007/0-387-23435-7_4. hal-02379886

\section{HAL Id: hal-02379886 https://hal.science/hal-02379886}

Submitted on 28 Nov 2019

HAL is a multi-disciplinary open access archive for the deposit and dissemination of scientific research documents, whether they are published or not. The documents may come from teaching and research institutions in France or abroad, or from public or private research centers.
L'archive ouverte pluridisciplinaire HAL, est destinée au dépôt et à la diffusion de documents scientifiques de niveau recherche, publiés ou non, émanant des établissements d'enseignement et de recherche français ou étrangers, des laboratoires publics ou privés. 


\title{
Chapter 3
}

\section{TRANSPOSING COMPUTER TOOLS FROM THE MATHEMATICAL SCIENCES INTO TEACHING.} Some possible obstacles.

\author{
Jean-Baptiste LAGRANGE \\ IUFM de Reims \& DIDIREM, Université Paris 7
}

\begin{abstract}
Thanks to the work of mathematicians, software designers and mathematics educators, computer algebra is now available on calculators that students can afford for classroom use. These new artifacts certainly open up stimulating prospects, but we should not look on them just as a miraculous solution to difficulties of teaching. We ought to initiate an in-depth reflection on their educational use in relation to the wider evolution of mathematics.

In this chapter, we will discuss how the new tools offered to students fit into the evolution of mathematics itself, and of mathematics teaching and learning. We will also consider difficulties in adapting teaching which often make integrating these new tools something of an adventure.
\end{abstract}

Key words: Transposition, Mathematical Sciences, Algorithms, Experimentation.

\section{THE FUTURE OF MATHEMATICS TEACHING}

In recent years there has been much discussion in France about the future of mathematics teaching (chapter 1). An official committee (the CREM, Commission de Réflexion sur l'Enseignement des Mathématiques) was created to think about what this future should be. According to this committee, a dramatic change has occurred over the past 50 years; mathematics is now produced and used by a great variety of people. One consequence is that we have to change our conception of mathematics to consider "the mathematical sciences" which are not just the concern of mathematicians.

The impact of ICT on mathematics teaching was a central issue in the discussion. The CREM committee devoted a report to this topic. According 
to this report, as ICT pervades all of society, mathematics is to be found everywhere in modern life, but people have no means to perceive this. Thus mathematics teaching should aim to make people aware of this hidden mathematics. ICT has also changed mathematics itself as the mathematical sciences now include an experimental dimension helped by the wide use of mathematical software.

According to the CREM, the use of mathematical software could help mathematics education to adapt to this new situation. A drawback is the sophistication of the mathematical software proposed for students' use, contrasting with the conceptual simplicity and clarity of traditional mathematics teaching According to the CREM, the possibility of achieving a similar simplicity and clarity should be secured by teaching basic ideas of data processing.

Box 3-1.

Extract from the CREM report (Kahane 2002).

In the recent evolution of mathematics, many new sources and outputs have appeared, as well as considerable work on existing mathematics. Other sciences and practices have provided mathematics with new problems, methods and concepts. New concepts and theories have been created and have sometimes proved useful in unexpected applications. Simulations based on mathematical models are present in every scientific activity and the development of mathematics benefits from both internal and external interaction. Thus mathematics is far from being just a matter for mathematicians. Contemporary mathematics can be described as a pumping, distilling and irrigating process involving physicists, computer scientists, engineers, biologists and economists together with mathematicians in the strict sense of the word.

As the CREM noted, curricula have recognized a need for such teaching but never really implemented it because of a lack of curricular contents and classroom activities. According to the CREM new prospects are opened up by the use of mathematical software, provided that teaching can clarify for students how mathematical data is represented and processed. Thus, basic notions about data representation and processing should be studied, together with numerical and symbolic experimental processes, number representation and processing, induction, graphs, convexity, continued fractions... This could provide the framework for a new algorithmic way of thinking in the common culture. 


\section{INTEGRATING NEW TOOLS: A PROBLEM OF TRANSPOSITION}

The goal of this book -conceptualizing the integration of symbolic calculators- is consistent with the above concern. The numerical, graphic, symbolic and programming capabilities of the new calculators to be found at school level are a transposition of computerized tools used in mathematical sciences. Thus they can play a major role in the future of mathematics teaching, not just as pedagogical aids but as a vehicle for new approaches. However, their use could conceal the mathematical basis of these approaches if teaching does not adapt its goals, contents and methods.

In this chapter, we will take advantage of didactical theorization and classroom observation to better understand these new approaches, and to look into the way in which teaching could be adapted. First we will focus on the global question of the impact of "the mathematical sciences" on teaching, taking into account that the purposes of professional mathematicians and researchers differ from the aims of teaching. We will use the notion of "didactical transposition". When he introduced this notion Chevallard (1985 p.14) stressed that "what happens inside a didactical system cannot be understood without considering what happens outside". He proposed to consider "genesis, filiations, gaps and reorganizations" interrelating mathematics teaching and professional mathematics. In this approach, mathematics in research and in school can be seen as a set of knowledge and practices in transposition between two institutions, the first one aiming at the production of knowledge and the other at its study ${ }^{2}$. French curricula clearly consider this prospect when they stress the role of the calculator in helping experimental approaches and the use of spreadsheets or calculators in carrying out algorithms (Box 1-1, Box 3-2). Filiations clearly appear but there must be hidden obstacles because this prospect was never really implemented in classrooms.

We see a cause of these obstacles in the different aims of the two institutions. Professional mathematics favors new approaches and reorganizations on the basis of productivity and mathematical correctness. In official research fields, data and methods to obtain conjectures using mathematical software are now being published and discussed. Some mathematicians specialize in the production and publication of experimental outcomes, while other mathematicians use these conjectures to work on proofs.

Experimental mathematics is that branch of mathematics that concerns itself ultimately with codification and transmission of insights within the mathematical community through the use of experimental exploration of 
conjectures and more informal beliefs and a careful analysis of the data acquired in this pursuit (Borwein et al. 1996).

In addition, computer science techniques in research motivate fundamental mathematical work about algorithms. Effectiveness -existence of an algorithm to solve a given problem-, complexity -the algorithm's properties in relation to processing time and data size- and efficiency -the practical conditions for the use of the algorithm on a given technology- are important notions in this work (Rouiller, Roy 2001, p. 35).

In contrast, teaching, especially in the general (rather than vocational) stream, is not primarily interested in improving mathematical productivity by way of new tools but rather in the transmission of a mathematical culture. The kernel of this culture lies in the social expectations of parents, students and teachers, and generally does not change easily. In order to survive in contemporary societies where ICT has a major role, this kernel should now integrate the potential of new tools and mathematical activity inspired by the mathematical sciences. Because the kernel was built when only traditional tools existed, this integration has a cost -a not-obvious in-depth reorganization- and resistance can be expected. To look at this reorganization, we will distinguish two dimensions, one about algorithms and the other about experimental approaches. These dimensions are certainly closely interrelated in the mathematical sciences, but, as we shall see, obstacles to their integration into teaching and learning are dissimilar.

\section{ALGORITHMS IN MATHEMATICS TEACHING}

Curricula have recommended the use of "programmable" calculators for fifteen years. A study of textbooks and practices shows that only graphic and numerical capabilities have actually been used in classrooms. More recently, the Euclidean algorithm for the greatest common divisor appeared at the $9^{\text {th }}$ grade and prime number search algorithms were introduced in the $12^{\text {th }}$ grade. To look into the difficulties of transposition we will consider the $9^{\text {th }}$ grade curriculum, analyzing how an algorithmic idea was transposed from advanced mathematics and how it was (mis)understood in textbooks. 
Box 3-2.

\begin{tabular}{|c|c|c|}
\hline \multicolumn{3}{|c|}{ Algorithmic approach in the French grade 9 curriculum } \\
\hline Content & Intended proficiency & Comments \\
\hline $\begin{array}{l}\cdots \\
4 \text { - Integer and } \\
\text { rational numbers. } \\
\text { Common divisors. } \\
\text { Irreducible } \\
\text { fractions. }\end{array}$ & $\begin{array}{l}\text { Being able to find whether } \\
\text { two given numbers have a } \\
\text { common divisor greater } \\
\text { than one. } \\
\ldots \\
\text { Being able to simplify a } \\
\text { given fraction into an } \\
\text { irreducible fraction. }\end{array}$ & $\begin{array}{l}\text {... The sum and the difference of two } \\
\text { multiples of an integer are themselves } \\
\text { multiples of this integer. It is then possible } \\
\text { to build an algorithm (Euclid's or } \\
\text { another). This algorithm will give the } \\
\text { GCD of two integers and answer the } \\
\text { question [of knowing whether a fraction is } \\
\text { irreducible]... Teaching will take } \\
\text { advantage of spreadsheets and CAS for } \\
\text { this topic. }\end{array}$ \\
\hline
\end{tabular}

The curriculum (Box 3-2) introduces the Euclidean algorithm in terms of two dimensions. The first one is theoretical: a fundamental property of divisors helps to build the algorithm. The second dimension is practical: offering the algorithm as means to recognize and obtain irreducible fractions. In addition, this dimension helps to insert the algorithmic approach inside the 'usual' mathematics. From the study of these two dimensions, mathematical and algorithmic meaning can be expected.

This introduction is consistent with the approach to algorithms current in advanced mathematics. For instance, the extracts from a computer science classic (Box 3-3) show that, at this level, the Euclidean algorithm helps to understand the notion of data processing (chapter 0), and to introduce a method for the formulation of algorithms (chapter 7). On one hand, the idea of transposing this approach into secondary teaching is stimulating because the algorithm appears to derive logically from properties of the GCD and from mathematically expressed constraints of execution (decrease of $x+y$ ). Emphasizing the links between an algorithm and the underlying mathematical properties is certainly something interesting to transpose into secondary education. On the other hand, the method deals with difficult logical concepts like weakest precondition and weakest condition such that the execution is guaranteed to decrease a function. 
Box 3-3.

\section{Euclid's algorithm in a computer science classic (Dijkstra 1976)}

Chapter 0: Executional abstraction

Let us consider a mechanism. On a cardboard with grid points, the only numbers written are the values 1 through 500 along both axes. The "answer line" with the equation $x=y$ is drawn. When we wish to find the greatest common divisor of two values $X$ and $Y$, we place a pebble on the grid point with the coordinates $x=X$ and $y=Y$. As long as the pebble is not lying on the "answer line", we consider the smallest ?equilateral rectangular triangle with its right angle coinciding with the pebble and one sharp angle on one of the axes. The pebble is then moved to the grid point coinciding with the other sharp angle of the triangle. The above move is repeated as long as the pebble has not yet arrived on the answer line.

Chapter 7: Euclid's algorithm revisited

... I shall now devote yet another chapter to Euclid's algorithm. I expect that in the meantime some of my readers will already have coded it in the form

$(x, y):=(X, Y)$;

do $\quad x>y \rightarrow x:=x-y ; y>x \rightarrow y:=y-x$ od;

$\operatorname{print}(x)$

Let us now try to forget the cardboard game and let us try to invent Euclid's algorithm afresh (...) Collecting our knowledge we can write down :

$G C D(X, Y)=G C D(Y, X)$

$G C D(X, Y)=G C D(X, X+Y)=G C D(X, X-Y)$

$G C D(X, Y)=a b s(X)$ if $X=Y$

...This is strongly suggestive of an algorithm that establishes the truth of $P=(G C D(X, Y)=G C D(x, y)$ and $x>0$ and $y>0) \ldots$ whereafter we 'massage' $(x, y)$ in such way that the relation $P$ is kept invariant. If we can manage this massaging process so as to reach a stage satisfying $x=y$, then we have found our answer by taking the absolute value of $x(\ldots)$

For the assignment $x:=x-y$ we find the weakest condition such that the execution is guaranteed to decrease $x+y$ is $y>0$, a condition that is implied by $P$.

Full of hope, we investigate the weakest precondition in order that $\mathrm{P}$ is valid after the assignment $x:=x-y$. (We find) $G C D(X, Y)=G C D(x-y, y)$ and $x-y>0$ and $y>0$. The outmost terms can be dropped as they are implied by $P$ and we are left with the middle one.

Thus we find $x>y->x:=x-y$ and $x>y->x:=x-y \ldots$ 
Box 3-4.

Euclid's algorithm in a $9^{\text {th }}$ grade textbook (Chapiron $\&$ al. 1999)

A method using iterated subtractions helps to find the Greatest Common Divisor of two numbers. This method is very old and known as Euclid's algorithm. The flow chart [below] explains how it works. Calculations are easy but repetitive and sometimes long. A spreadsheet helps to calculate more quickly.

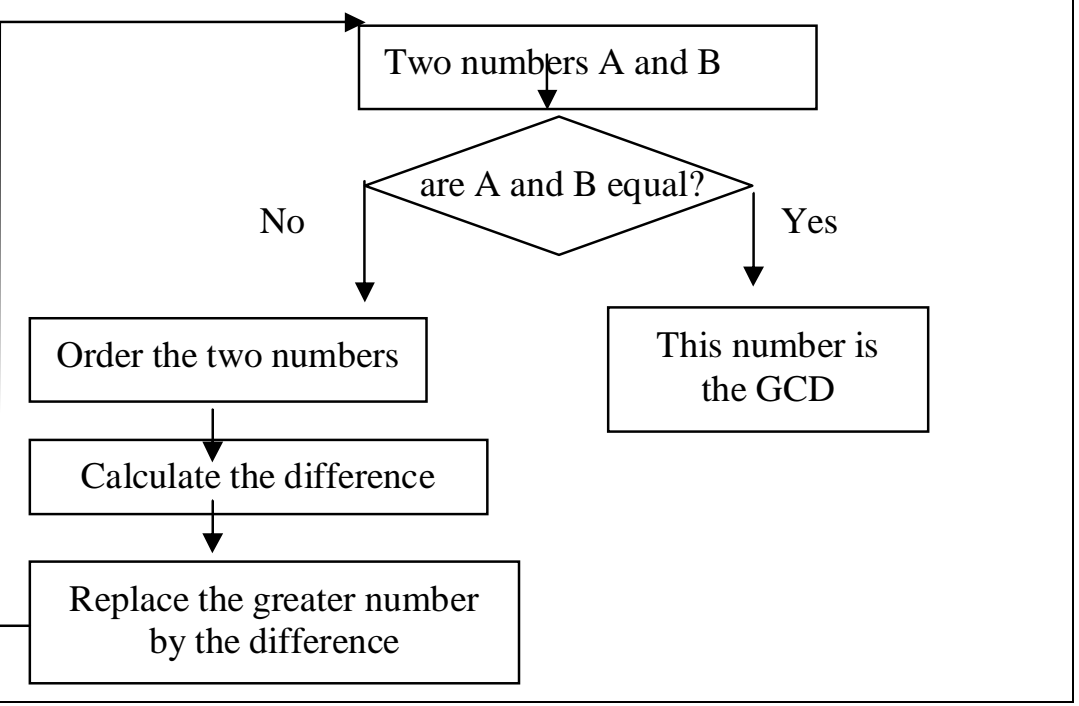

It appears that the curriculum considered the interest of the method but not the difficulties of its existence as an isolated object. Proof of this can be found in the way in which textbooks (mis)interpret the curriculum. In contrast with the curriculum and Dijkstra's book, a typical textbook (Box 3-4) presents neither practical nor theoretical dimensions of Euclid's algorithm. It offers just a flow chart and a 'push button' translation into a spreadsheet. Other textbooks also adopt this approach. The reason why textbooks did not follow the curriculum when it offered the algorithm for practical simplification of fractions is that they judged that this use is not realistic. The logical difficulties of constructing the algorithm from properties of the GCD and the constraints of execution certainly explain why textbooks offer no theoretical dimension. Obviously, the curriculum tried to introduce Euclid's algorithm without much change in the more general background. Under these conditions the algorithm is an isolated object without a real mathematical existence, and textbooks are not able to make something interesting of this object.

Introducing a real algorithmic dimension by using calculators in mathematics teaching and learning would imply greater change. But, then, another difficulty would be that there is very little didactical research able to 
offer help in thinking about this change. Research about the learning of algorithmic processing tends to be old and isolated. Rogalski \& Samurçay (1990) found important conceptual difficulties, even with very simple iterative processes, but no other research studies followed. There are also too few research studies about data representation (Aharoni 2000 is a very isolated example). The case of the algorithmic approach shows that, even when powerful tools are available, we remain a long way short of achieving a cultural algorithmic way of thinking, through a satisfactory transposition of advanced mathematics to teaching and learning in the secondary school.

\section{EXPERIMENTAL APPROACHES IN TEACHING AND LEARNING}

As compared to algorithms, the experimental dimension of mathematical sciences seems at first sight more easy to transpose into the kernel of mathematical culture, as mentioned above. In mathematical research, producing and trying conjectures helps to discover new theorems and to build new theories. Experimental approaches cannot have this role in teaching, but other contributions are generally expected. Authors like Péres (1998) and Kutzler (1997) start from the idea that experimenting can help students to develop a more in-depth understanding of mathematics. They generally stress that 'traditional teaching' does not work properly because students have just to repeat routines and are not allowed to search by themselves. In these authors' view, experimenting with new tools will be a remedy. For instance, students, even with weak abilities in arithmetic or algebraic procedures, might be able to use symbolic calculators to explore advanced mathematical domains or to try several approaches to problems that they could not do by hand. Thus, with new tools, mathematical teaching would become more interesting and accessible to more students.

On one hand, using the potentialities of new tools in experimental approaches is a stimulating idea. The visualizing capabilities of computers could help more varied access to mathematical problems and concepts. Exploring a problem, students could study a number of examples, using varied representations of objects and inductive as well as deductive approaches. On the other hand, in learning, as in mathematical sciences, understanding of concepts does not emerge spontaneously from observation, even with the help of powerful tools.

Experimental approaches in research are harnessed to the production of new knowledge. This articulation is what Borwein \& al. (ibid. p. 16) named "theoretical experimentation" and includes structuring a domain to formulate hypotheses, deriving examples to try with a machine, interpreting the 
machine results... Transposition should thus maintain the linking of experimentation with theoretical elaboration. Reflecting on this transposition at the beginning of the TI-92 experiment, we found that, in teaching and learning, this articulation is far from obvious, and that authors stressing the potentialities of new tools generally tended to underestimate the difficulties of their classroom use. To explain this, let us look at classroom situations involving an experimental activity.

We consider two classes of situations. In the first class, students have to observe and interpret a number of calculator answers. For instance, approaching multiple representations of a concept, students have to consider perceptually how a mathematical property appears as a phenomenon in several windows of a calculator; or in an applied mathematics course, students are meant to use a CAS to avoid too complicated hand calculation. In the second class of situations students have to experiment on symbolic phenomena and find general structures by induction. In other words, students have to observe 'how the machine does it', try to discover techniques 'to do the same as the machine' and give a mathematical interpretation of the machine operation. The two classes of situations differ in terms of the consideration given to the machine operation. In the first class, a student is expected just to use the computer output as mathematically relevant results, whereas in the second (s)he is meant to think about how they were obtained.

Berry \& al (1994) identified "five potential ways to integrate the use of DERIVE into a mathematical course" (Box 3-5). The first class of situation that we consider in this chapter corresponds to potential way 2 and 5 ("as a problem solving assistant" and "an aid to visualization and interpretation") and the second class corresponds to potential way 3 ("as an investigative or exploratory environment").

Let us consider a situation from the first class, related to the idea of function. To help students to approach this idea, it is important to provide them with varied views on the relationship between representations. For instance one view of the relationship between algebraic definition and graphic representation arises through considering that a zero of the function corresponds to an intersection point between the graphic representation and the $\mathrm{x}$-axis. It is not a spontaneous view because, at first, students see the intersection's coordinates as the solution of a two equation system. To help students to interpret graphically the zeros of a function, it is interesting to ask them to consider together these values -obtained by algebraic solutionand the coordinates of the intersection points -read from the graph. Using symbolic calculators is helpful because the two operations are performed by specific commands in two separate windows. 
Box 3-5.

Classroom situations involving an experimental activity.

(Berry \& al 1994)

DERIVE as a problem solving assistant (p. 84)

In many mathematical modeling courses greater emphasis is placed on the formulation of problems and the interpretation of results, rather than the solution of the mathematical problems that may occur. Similarly, courses in applied mathematics may wish to concentrate more on the concepts and relationships that form the basis of the study but may also include extensive use of algebraic manipulation or calculus. In the past the emphasis in these types of courses has become distorted due to the large amount of time spent by the students obtaining solutions to problems compared to the important formulation and interpretation stages (...) By reducing the time that students need to spend obtaining solutions and increasing their reliability and their accuracy, DERIVE allows more emphasis to be placed on the formulation and interpretation phases of mathematical modeling or applied mathematics. In particular it allows students to use mathematical concepts and techniques that they understand in principle, can apply in simple cases, but find difficult to apply in the more complex cases that may arise in real problem solving.

DERIVE as an aid to visualization and interpretation (p. 93)

It is important for students to be able to visualize and interpret mathematical results. Often with some higher level mathematics it is quite hard for students to do so and DERIVE can offer an environment in which it is possible to do mathematics and create visual images that allow students to interpret and comment on the results they have obtained. A student may show that a Maclaurin series approximation to $\sin x$ is $x-x^{3} / 6$. It is quite hard however for the student to relate this back to the original function, or establish the range of values over which the approximation is reasonable. It is very simple to produce a series approximation of this type on DERIVE and compare its plot with a plot of the original function.

DERIVE as an investigative or exploratory environment (p. 86)

It is possible to use DERIVE as an environment in which students can exploit and learn new mathematics by making discoveries for themselves(...) One very real benefit of this approach is that the students can gain an intuitive feel for mathematical ideas and principles before they receive a formal introduction to the mathematics. As an example consider the chain and product rules for differentiation. A typical text book introduction would give either a formal statement or derivation of the rule followed by worked examples. Alternative approaches designed to develop in students an initial intuitive feel for these rules have been laborious for students to carry out. DERIVE however offers the potential to develop these intuitive ideas very easily.

Situations of this type are often presented to promote the use of new tools, but it is seldom mentioned that they are effective only if students have a suitable preparation. Wain (1994) reports on an observation of students not able to recognize the decimal value that they read in the graphic window as an approximation of the symbolic solution that they obtained in the algebraic 
window. In contrast with the teacher's expectation, they could not see a relationship between zeros and intersection points.

Our opinion is that, before experiencing this situation, students should have been prepared to recognize the differing form taken by a number under several types of expression, particularly exact symbolic expressions in the symbolic window and approximate values in the tabular or graphic window. Delgoulet \& Guin (1997) designed and experimented with classroom activities to achieve this preparation. See chapter $9 \S 2$.

Box 3-6 provides another example of a situation where it was assumed that CAS use would be transparent to students. DERIVE was supposed to help students to acquire better methods of transforming trigonometric expressions by performing automatically the more technical part of the transformation. But it failed because of students' misunderstanding of what a simplification process involves for a machine. Ruthven's analysis put to the fore the 'sense of the command' that would be necessary. Even when CAS is 'just a tool' an understanding of its technical operation cannot be avoided.

In the second class of classroom situations, symbolic capabilities are means for students to carry out algebraic transformations before knowing how to perform paper/pencil techniques and even before knowing the existence of these techniques and their mathematical underpinning. After a first encounter with notions like limits or derivatives, students could use a symbolic calculator to experiment with symbolic transformations (limits or derivatives of sums and products, for instance) and become aware of algebraic rules (or algebraic techniques) applied by the machine. They could then imagine general structures underpinning these rules.

This use of computer tools is also a stimulating prospect but we have to be aware that for students, detecting symbolic phenomena and inductively formulating algebraic rules and techniques might not be so obvious. During the TI-92 calculator experiment (chapter 9) we had to reflect on how an experimental inductive activity about symbolic techniques for limits and derivatives could really be made to work.

Our first concern was to find questions that could provoke students' inductive reflection. Questions like 'observe what happens' do not necessarily yield interesting observations. Many algebraic transformations actually maintain the structure of most expressions -for instance when the sum of limits is the limit of the sum- and such examples are, of course, not problematic. Even when the structure is not maintained -for instance in the case of indefinite limits or of differentiation of a product- students do not spontaneously start thinking inductively. Results that the teacher expected to be amazing to students (for instance DERIVE simplifications for the chain and product rules of differentiation in Box 3-5) did not alone create much surprise. The learning situation has to bring to the fore puzzling peculiarities 
and to challenge students' anticipations. For instance it is often interesting to prepare examples where students' predictions will very probably be wrong and to ask them to compare these predictions with the calculator's answer.

Box 3-6.

Difficulties with emergent subgoals. The task of transforming the trigonometric sum, $\sin (x)+\sin (2 x)$, into a trigonometric product.

(Ruthven 2002, pp. 287-8)

The French research provides a specific example in an episode in which students - relatively experienced in using CAS - were charged with the task of transforming the trigonometric sum, $\sin (x)+\sin (2 x)$, into a trigonometric product (Lagrange 2000, p. 5).

In response to a programmed command, the CAS gave the expression $2 \sin ((2 x+x) / 2) \cos ((2 x-x) / 2)$. The students wanted to simplify this to $2 \sin (3 x / 2) \cos (x / 2)$. To their surprise, the response of the CAS to repeated simplify commands was first to give the original expression and then $\sin (x)+2 \sin (x) \cos (x)$. To the students, aware of the overarching goal, the emergent subgoal of simplifying the subsidiary algebraic expressions $2 x+x$ and $2 x-x$ was clear. To them, this was transparently the sense of the command to simplify. In other words, their articulation of the simplification operation was a situated one. But, of course, no model of the larger task - and no situated sense of the command - was available to the CAS; it was unable to take account of the wider mathematical context giving rise to the instruction. The machine is unable to interpret or adapt an instruction to accord with the wider purpose so evident to a user; it can only operate literally, either in terms of the formal elections made by the user, or of preset defaults - which, as in this case, may fail to coincide serendipitously with the wider purpose of the user. The effective instrumentation of mathematical reasoning by means of a CAS depends, then, on precise reframing of situated purposive actions into the decontextualised formal register of the machine, and a corresponding reframing of results.

A second concern was the knowledge that students need in order to understand observations as phenomena within an inductive approach, to construct interesting examples, and to interpret the calculator's answers correctly. An example from Pozzi (1994) will help in examining this question. Pozzi reports on an observation of two students who were trying to find a general rule for differentiating a product by observing how DERIVE computes derivatives of the product of a polynomial with a trigonometric function. Asking DERIVE to differentiate $\cos (x)\left(7 x^{3}+2 x\right)$, they got $\left(21 x^{2}+2\right) \cos (x)-x\left(7 x^{2}+2\right) \sin (x)$. They then concentrated on the central part, $\cos (x)-x\left(7 x^{2}+2\right)$, which they found very similar to the initial expression. They tried to induce a general rule involving the transformation of a product into a difference. Of course the central part has no meaning because it is not a sub-expression of the derivative. But, to students, it appeared to be the key 
to finding a rule because it is perceptually close to the initial expression. Students' algebraic knowledge about the structure of expressions was not strong enough to counter balance this perceptual evidence and they could not make good use of DERIVE's help.

Thus, considering new tools as providing 'scaffolding' (Kutzler, 1997) for weak student knowledge is an idea which needs to be re-examined. From Pozzi's example it is clear that we have to reflect on the prior algebraic knowledge required. Students do not necessarily need strong procedural abilities but obviously should not be lacking some key knowledge of algebraic structure (chapter 7). Finally we had another concern about knowledge of the target concept itself in relation to the machine operation. For instance if students follow an experimental approach to a concept like limit mainly using the transformational capabilities of CAS, they will then associate the concept too closely with transformational rules and/or develop a 'push button' conception of the concept. Other modifications to the meaning of concepts may result from computer implementation and also interfere with experimental computer aided activity (chapter 5).

\section{CONCLUSION}

This chapter started from the idea of transposing approaches from the mathematical sciences into teaching and learning as a major avenue through which to make sense of the use of new computer tools. We have seen that this is not so easy. In particular, a real teaching of algorithms is not feasible today because the traditional cultural kernel underpinning curricula is resistant, and too few research studies and experiments have been undertaken. Transposition of experimental approaches seems more viable, but difficulties are very often underestimated. We located obstacles to computer aided experimental activity making an intended contribution to conceptualization. It appears that using computer symbolic tools as resources in perceptual and inductive approaches requires reflection on what prior knowledge students need both in algebra and about the machine, on what questions can serve to provoke inductive thinking, and on what form students' representation of concepts and of the machine operation takes.

The consequence is that experimental computer-aided approaches to teaching and learning cannot be thought of as simply a matter of using a machine to ease problem solving or to enhance inductive activity. Following Ruthven (2002), using CAS for graphic and symbolic reasoning "influences the range and form of the tasks and techniques ${ }^{3}$ experienced by students", and because tasks and techniques are resources available for more explicit codification it also influences "the theorization of such reasoning". Chapter 4 
will give an example where teachers participating in a common project take radically different and somehow restricting options regarding management of this phenomenon in the classroom. Chapter 5 will provide further insight both practical and theoretical. Task and technique will be offered as structural levels organizing the study of a mathematical domain and connecting experimental approaches to graphic and symbolic problem solving with conceptual elaboration.

$* * * * * * * * * * * * * * * * * * * * * * * * * *$

Jean-Baptiste Lagrange is a Professor at the Institut Universitaire de Formation des Maîtres in Reims.

He has contributed to the development of CAS use in schools and to the associated didactical reflection. His present work includes the design of a CAS environment for teaching and learning at secondary-school level and investigation of the classroom implementation of this environment. He is also working to contribute to a better understanding of the professional situation of teachers trying to integrate ICT.

\footnotetext{
${ }^{1}$ Our translation.

${ }^{2}$ The notion of study is presented in Box 5.2.

${ }^{3}$ Our emphasis.
} 


\section{REFERENCES}

Aharoni D. (2000) What you see is what you get - the influence of visualization on the perception of data structures. Proceedings of the 24th conference of theInternational Group for the Psychology of Mathematics Education (PME). Hiroshima, Japan.

Berry J., Graham T. \& Watkins A. (1994) Integrating the DERIVE program into the teaching of Mathematics. The International DERIVE Journal, Vol. 1.1, pp. 83-96.

Borwein J., Borwein P., Girgensohn R. \& Parnes S. (1996) Making sense of Experimental Mathematics. The mathematical Intelligencer Vol 18 n 4 Springler, New York, pp. 12-17.

Chapiron G., Mante M., Mulet, R.\& Perotin C. (1999) Mathématiques. Collection Triangle. Hatier.

Chevallard Y. (1985) La transposition didactique, Editions La Pensée Sauvage, Grenoble.

Delgoulet J. \& Guin D. (1997) Etude des modes d'appropriation de calculatrices graphiques et symboliques dans une classe de Seconde. IREM de Montpellier.

Dijkstra, E. W. (1976) A discipline of programming. Prentice Hall.

Kahane, J. (2002) L'enseignement des sciences mathématiques. Centre National de Documentation Pédagogique, Odile Jacob, Paris.

Kutzler B. (1997) With the TI-92 Towards Computer Age Maths Teaching, International Journal of Computer lgebra in Mathematics Education, $\mathrm{n}^{\circ} 4.1$, pp. 7-32.

Lagrange, J.B. (2000) L'intégration d'instruments informatiques dans l'enseignement: une approche par les techniques. Educational Studies in Mathematics, 43.1, pp. 1-30

Pérez Fernández, J. (1998) Los systemas de cálculo simbl'olico en la enseñza de las matemáticas. In Hodgson et al. eds) Selected lectures 8th International Congress on Mathematical Education, SAEM THALES, p. 345-368.

Pozzi S. (1994) Algebraic Reasoning and CAS: Freeing Students from Syntax? In Heugl H., Kutzler B. (eds) DERIVE in Education, Chartwell-Bratt, Bromley.

Rogalski J., Samurcay R. (1990) Acquisition of Programming Knowledge ans Skills. In Hoc J.M., Green T.R.G., Samurçay R., Gilmore D.J. (eds) Psychology of Programming, Academic Press, pp. 157-170

Rouiller, F. \& Roy, M.F (2001) Calculer avec des inégalités. Introduction aux algorithmes de la géométrie algébrique réelle. In Lagrange, Lenne (eds) Calcul formel et apprentissage des mathématiques. INRP Paris France.

Ruthven, K. (2002) Instrumenting mathematical activity: Reflections on key studies of the Educational use of computer algebra systems International Journal of Computers for Mathematical Learning 7: pp. 275-291.

Wain G. (1994) Some Technical Problems in the Use of DERIVE with School Pupils. International DERIVE Journal, ${ }^{\circ} 1.1$, pp. 49-56 